\title{
Existence and Uniqueness of Positive Solutions for a Coupled System of Nonlinear Fractional Differential Equations
}

\author{
Minjie Li, Yiliang Liu \\ College of Sciences, Guangxi University for Nationalities, Nanning 530006, Guangxi Province, P. R. China \\ Email: minjieli1988@126.com, yiliangliu100@126.com
}

Received 2013

\begin{abstract}
In this paper, we research the existence and uniqueness of positive solutions for a coupled system of fractional differential equations. By means of some standard fixed point principles, some results on the existence and uniqueness of positive solutions for coupled systems are obtained.
\end{abstract}

Keywords: Caputo Fractional Derivative; Fractional Differential Equations; Coupled System; Fixed Point Theorem; Positive Solutions

\section{Introduction}

Fractional differential equations can describe many phenomena in various fields of engineering and scientific disciplines such as control theory, physics, chemistry, biology, economics, mechanics and electromagnetic. In recent years, there are a large number of papers dealing with the existence of positive solutions of boundary value problems for nonlinear differential equations of fractional order. We refer readers to the monographs such as Kilbas etal. [8], Miller and Ross [20], Podlubny [21], and the papers [1,3-5,12-19,28-33] and references therein.

In [12], Li, Luo and Zhou considered the existence of positive solutions of the following boundary value problem of fractional order differential equations:

$$
\begin{aligned}
& D_{0+}^{\alpha} u(t)+f(t, u(t))=0,0<t<1, \\
& u(0)=0, D_{0+}^{\beta} u(1)=a D_{0+}^{\beta} u(\xi),
\end{aligned}
$$

where $D_{0+}^{\alpha}$ is the standard Riemann-Liouville fractional derivative of order $1<\alpha \leq 2,0<\beta \leq 1,0 \leq a \leq 1, \xi \in(0,1)$, $a \xi^{\alpha-\beta-2} \leq 1-\beta, 0 \leq \alpha-\beta-1$ and $f:[0,1] \times[0, \infty) \rightarrow[0, \infty)$ satisfies Caratheodory type conditions.

In [30], Yang, Wei and Dong investigated the following existence of positive solutions of fractional order differential equations:

$$
\begin{aligned}
& { }^{c} D_{0+}^{\alpha} u(t)=f\left(t, u(t), u^{\prime}(t)\right), 0<t<1, \\
& u(0)+u^{\prime}(0)=0, u(1)+u^{\prime}(1)=0,
\end{aligned}
$$

where ${ }^{c} D_{0+}^{\alpha}$ is the Caputo fractional derivative of order $1<\alpha \leq 2$ and $f \in C\left([0,1] \times[0, \infty) \times R, R^{+}\right)$.
In addition, recently some authors also pay close attention to the existence of solutions for coupled systems of fractional differential equations (see[2,3,25,26]).

In [26], Su studied the existence of solutions for a coupled system of fractional differential equations:

$$
\left\{\begin{array}{l}
D_{0+}^{\alpha} u(t)=f\left(t, v(t), D_{0+}^{\mu} v(t)\right), 0<t<1, \\
D_{0+}^{\beta} v(t)=g\left(t, u(t), D_{0+}^{v} u(t)\right), 0<t<1, \\
u(0)=u(1)=v(0)=v(1),
\end{array}\right.
$$

where $1<\alpha, \beta<2, \mu, v>0, \alpha-v \geq 1, \beta-\mu \geq 1, f, g:[0,1] \times R^{2} \rightarrow R$ are given functions and $D_{0+}$ is the standard RiemannLiouville fractional derivative.

In [25], Sun, Liu and Liu considered the following systems of fractional differential equations with antiperiodic boundary conditions:

$$
\left\{\begin{array}{l}
{ }^{c} D_{0+}^{\alpha} u(t)=f_{1}\left(t, u(t), v(t),{ }^{c} D_{0+}^{p} u(t),{ }^{c} D_{0+}^{q} v(t)\right), \\
t \in J=[0, T], \\
{ }^{c} D_{0+}^{\beta} v(t)=f_{2}\left(t, u(t), v(t),{ }^{c} D_{0+}^{p} u(t),{ }^{c} D_{0+}^{q} v(t)\right), \\
t \in J=[0, T], \\
u(0)=-u(T), u^{\prime}(0)=-u^{\prime}(T), \\
v(0)=-v(T), v^{\prime}(0)=-v^{\prime}(T),
\end{array}\right.
$$

where ${ }^{c} D_{0+}^{\alpha}$ denotes the Caputo fractional derivative, $1<\alpha, \beta \leq 2,0<p, q<1, f_{1}, f_{2} \in C\left(J \times R^{4}, R\right)$.

However, the research on the systems of positive solutions of fractional differential equations hasn't received remarkable attention. In this paper, we shall concern with the existence and uniqueness of positive solutions for a cou- 
pled system of nonlinearfractional differential equations. More precisely, we will consider the following problem:

$$
\left\{\begin{array}{l}
{ }^{c} D_{0+}^{\alpha} u(t)=f_{1}\left(t, u(t), v(t),{ }^{c} D_{0+}^{p} u(t),{ }^{c} D_{0+}^{q} v(t)\right), \\
t \in J=[0, T], \\
{ }^{c} D_{0+}^{\beta} v(t)=f_{2}\left(t, u(t), v(t),{ }^{c} D_{0+}^{p} u(t),{ }^{c} D_{0+}^{q} v(t)\right), \\
t \in J=[0, T], \\
u(0)+\lambda_{1} u{ }^{\prime}(0)=0, \quad u(1)+\lambda_{2}{ }^{c} D_{0+}^{p} u(1)=0, \\
v(0)+\lambda_{1} v^{\prime}(0)=0, \quad v(1)+\lambda_{2}{ }^{c} D_{0+}^{q} v(1)=0,
\end{array}\right.
$$

where ${ }^{c} D_{0+}^{\gamma}$ denotes the Caputo fractional derivative for $\gamma>0,1<\alpha, \beta \leq 2,0<p, q \leq 1, f_{1}, f_{2} \in C\left([0,1] \times R_{+}^{2} \times R^{2}, R_{+}\right)$.

This paper is organized as follows: In section 2 , we introduce some preliminary results, including basic definitions of fractional integrals and derivatives, some properties and a fixed point theorems. In section 3, by applying some standard fixed point principles, we prove the existence and uniqueness of positive solutions for a coupled system of nonlinear fractional differential equations.

\section{Preliminaries}

Let us introduce a space: $X=\left\{u(t) \mid u(t) \in C^{1}([0,1])\right\}$ endowed with the norm

$$
\|u\|_{X}=\max _{t \in[0,1]}|u(t)|+\max _{t \in[0,1]}\left|u^{\prime}(t)\right| .
$$

Indeed, $\left(X,\|.\|_{X}\right)$ is a Banach space. Obviously, the product space $\left(X \times X,\|\cdot\|_{X \times X}\right)$ is also a Banach space with $\|(u, v)\|_{X \times X}=\|u\|_{X}+\|v\|_{X}$. For the convenience of the readers, we first present some useful definitions and fundamental facts of fractional calculus theory, which can be found in $[8,21]$.

Definition 2.1. For $\gamma>0$, the integral

$$
I_{0+}^{\gamma} f(t)=\frac{1}{\Gamma(\gamma)} \int_{0}^{t} \frac{f(s)}{(t-s)^{1-\gamma}} d s,
$$

is called the Riemann-Liouville fractional integral.

Definition 2.2. For a function $f(t)$ given in the interval $[0, \infty)$, the expression

$$
\begin{aligned}
& { }^{L} D_{0+}^{\gamma} f(t)=\frac{1}{\Gamma(n-\gamma)}\left(\frac{d}{d t}\right)^{n} \int_{0}^{t} \frac{f(s)}{(t-s)^{\gamma-n+1}} d s, \\
& n=[\gamma]+1
\end{aligned}
$$

is called the Riemann-Liouville fractional derivative of order $\gamma>0$, where $[\gamma]$ denotes the integer part of real number $\gamma$.

Definition 2.3 [6]. The Caputo's derivative of order $\gamma$ for a function $f \in C^{n}([0, \infty), R)$ can be written as

$$
\begin{aligned}
& { }^{c} D_{0+}^{\gamma} f(t)={ }^{L} D^{\gamma}\left[f(t)-\sum_{k=0}^{n-1} \frac{t^{k} f^{(k)}(0)}{k !}\right], \\
& n-1<\gamma<n .
\end{aligned}
$$

Lemma $2.4[8,21]$. Let $u \in C^{n-1}[0,1]$ and $q \in(n-1, n]$, $n \in N$. Then for $t \in[0,1]$,

$$
I^{q c} D_{0+}^{q} u(t)=u(t)-\sum_{k=0}^{n-1} \frac{t^{k}}{k !} u^{(k)}(0) .
$$

Lemma 2.5. Let $\varphi \in C[0,1]$. If $H: 1 \leq \lambda_{1}<1+\frac{\lambda_{2}}{\Gamma(2-p)}$, $\lambda_{2}>0$, then $u(t)$ is a solution of the following fractional differential equations:

$$
\left\{\begin{array}{l}
{ }^{c} D_{0+}^{\alpha} u(t)=\varphi(t), t \in[0,1], 1<\alpha<2, \\
u(0)+\lambda_{1} u^{\prime}(0)=0, u(1)+\lambda_{2}^{c} D_{0+}^{p} u(1)=0,0<p<1,
\end{array}\right.
$$

if and only if $u(t)$ is a solution of the fractional integral equations

$$
u(t)=\int_{0}^{1} G_{\alpha}(t, s) \varphi(s) d s
$$

where

$$
\begin{aligned}
& G_{\alpha}(t, s)=\left\{\begin{array}{l}
\frac{\Lambda(t-s)^{\alpha-1}+\left(\lambda_{1}-t\right)(1-s)^{\alpha-1}}{\Lambda \Gamma(\alpha)} \\
+\frac{\lambda_{2}\left(\lambda_{1}-t\right)(1-s)^{\alpha-p-1}}{\Lambda \Gamma(\alpha-p)}, \\
0 \leq s \leq t \leq 1, \\
\frac{\left(\lambda_{1}-t\right)(1-s)^{\alpha-1}}{\Lambda \Gamma(\alpha)}+\frac{\lambda_{2}\left(\lambda_{1}-t\right)(1-s)^{\alpha-p-1}}{\Lambda \Gamma(\alpha-p)} \\
0 \leq t \leq s \leq 1,
\end{array}\right. \\
& \Lambda=1+\frac{\lambda_{2}}{\Gamma(2-p)}-\lambda_{1} .
\end{aligned}
$$

Furthermore, if the assumption $H$ holds, then $G_{\alpha}(t, s) \in C([0,1) \times[0,1))$ and $G_{\alpha}(t, s)>0$, for any $t, s \in(0,1)$.

Proof. Assume $u(t)$ satisfies (2.5). By (2.4), (2.5), we have

$$
u(t)=\int_{0}^{t} \frac{(t-s)^{\alpha-1}}{\Gamma(\alpha)} \varphi(s) d s+u(0)+u^{\prime}(0) t .
$$

Hence,

$$
u^{\prime}(t)=\int_{0}^{t} \frac{(t-s)^{\alpha-2}}{\Gamma(\alpha-1)} \varphi(s) d s+u^{\prime}(0)
$$

By definition 2.3 together with the facts that

$$
{ }^{L} D_{0+}^{p} t=\frac{t^{1-p}}{\Gamma(2-p)}, \quad{ }^{L} D_{0+}^{p} I^{\alpha} u(t)=I^{\alpha-p} u(t)
$$

and the linearity of fractional differential, we get

$$
{ }^{c} D_{0+}^{p} u(t)=\int_{0}^{t} \frac{(t-s)^{\alpha-p-1}}{\Gamma(\alpha-p)} \varphi(s) d s+u^{\prime}(0) \frac{t^{1-p}}{\Gamma(2-p)} .
$$

Applying the boundary conditions

$$
u(0)+\lambda_{1} u^{\prime}(0)=0, u(1)+\lambda_{2}^{c} D_{0+}^{p} u(1)=0,
$$

we obtain that 


$$
\begin{aligned}
u(0)= & \frac{\lambda_{1}}{\Lambda \Gamma(\alpha)} \int_{0}^{1}(1-s)^{\alpha-1} \varphi(s) d s+\frac{\lambda_{1} \lambda_{2}}{\Lambda \Gamma(\alpha-p)} \\
& \times \int_{0}^{1}(1-s)^{\alpha-p-1} \varphi(s) d s, \\
u^{\prime}(0)= & -\frac{1}{\Lambda \Gamma(\alpha)} \int_{0}^{1}(1-s)^{\alpha-1} \varphi(s) d s-\frac{\lambda_{2}}{\Lambda \Gamma(\alpha-p)} \\
& \times \int_{0}^{1}(1-s)^{\alpha-p-1} \varphi(s) d s .
\end{aligned}
$$

Consequently,

$$
\begin{aligned}
u(t)= & \int_{0}^{t} \frac{(t-s)^{\alpha-1}}{\Gamma(\alpha)} \varphi(s) d s+\frac{\lambda_{1}-t}{\Lambda \Gamma(\alpha)} \int_{0}^{1}(1-s)^{\alpha-1} \varphi(s) d s \\
& +\frac{\lambda_{2}\left(\lambda_{1}-t\right)}{\Lambda \Gamma(\alpha-p)} \int_{0}^{1}(1-s)^{\alpha-p-1} \varphi(s) d s \\
= & \int_{0}^{1} G_{\alpha}(t, s) \varphi(s) d s .
\end{aligned}
$$

Conversely, assume $u(t)$ is a solution of fractional integral equations (2.6). Using the definition of Caputo's derivative (2.3) and the fact that ${ }^{L} D_{0+}^{p} C=\frac{C t^{2-p}}{\Gamma(1-p)}$ and ${ }^{L} D_{0+}^{\alpha}$ is the left inverse of $I_{0+}^{\alpha}$ we get (2.5).

Observing the expression of $G_{\alpha}(t, s)$ in (2.6), we easily obtain $G_{\alpha}(t, s) \in C([0,1) \times[0,1))$. Let

$$
\begin{aligned}
& g_{1}(t, s)= \frac{(t-s)^{\alpha-1}}{\Gamma(\alpha)}+\frac{\left(\lambda_{1}-t\right)(1-s)^{\alpha-1}}{\Lambda \Gamma(\alpha)} \\
&+\frac{\lambda_{2}\left(\lambda_{1}-t\right)(1-s)^{\alpha-p-1}}{\Lambda \Gamma(\alpha-p)}, 0 \leq s \leq t \leq 1, \\
& g_{2}(t, s)= \frac{\left(\lambda_{1}-t\right)(1-s)^{\alpha-1}}{\Lambda \Gamma(\alpha)}+\frac{\lambda_{2}\left(\lambda_{1}-t\right)(1-s)^{\alpha-p-1}}{\Lambda \Gamma(\alpha-p)}, \\
& 0 \leq t \leq s \leq 1
\end{aligned}
$$

By $H$, we have $\Lambda>0$ and

$$
\begin{aligned}
& g_{2}(t, s)=\frac{\left(\lambda_{1}-t\right)(1-s)^{\alpha-1}}{\Lambda \Gamma(\alpha)}+\frac{\lambda_{2}\left(\lambda_{1}-t\right)(1-s)^{\alpha-p-1}}{\Lambda \Gamma(\alpha-p)}>0, \\
& \forall s, t \in(0,1),
\end{aligned}
$$

which also implies $g_{1}(t, s)>0$ by (2.8). Hence $G_{\alpha}(t, s)>0$ for all $s, t \in(0,1)$. The proof is completed.

Remark 2.6. If we make use of

$$
H^{\prime}: \lambda_{1} \geq 1, \lambda_{2}<-\frac{\Gamma(\alpha-p)}{\Gamma(\alpha)}
$$

instead of $H$, we may similarly consider the problem (1.1). We omit it here.

Lemma 2.7 [22]. Let $E$ be a Banach space. Assume that $T: E \rightarrow E$ is a completely continuous operator and the set $V=\{u \in E \mid u=\mu T u, 0 \leq \mu \leq 1\}$ is bounded. Then $T$ has a fixed point in $E$.

\section{Main Result}

For the sake of convenience, we set

$$
\begin{gathered}
M_{1}=\frac{\Lambda+\lambda_{1}+\Lambda \alpha+1}{\Lambda \Gamma(\alpha+1)}+\frac{\lambda_{2}\left(\lambda_{1}+1\right)}{\Lambda \Gamma(\alpha-p+1)}, \\
M_{2}=\frac{\Lambda^{*}+\lambda_{1}^{*}+\Lambda^{*} \alpha+1}{\Lambda^{*} \Gamma(\alpha+1)}+\frac{\lambda_{2}^{*}\left(\lambda_{1}^{*}+1\right)}{\Lambda^{*} \Gamma(\alpha-p+1)}, \\
M_{3}=\max \left\{\left[M_{1} c_{1}+M_{2} d_{1}+\left(M_{1} c_{3}+M_{2} d_{3}\right) \frac{1}{\Gamma(2-p)}\right],\right. \\
\left.\left[M_{1} c_{2}+M_{2} d_{2}+\left(M_{1} c_{4}+M_{2} d_{4}\right) \frac{1}{\Gamma(2-q)}\right]\right\} .
\end{gathered}
$$

We denote

$$
\Lambda=1+\frac{\lambda_{2}}{\Gamma(2-p)}-\lambda_{1}, \Lambda^{*}=1+\frac{\lambda_{2}^{*}}{\Gamma(2-q)}-\lambda_{1}^{*}
$$

and give the following assumption

$$
\begin{aligned}
& H^{*}: 1 \leq \lambda_{1}<1+\frac{\lambda_{2}}{\Gamma(2-p)}, \lambda_{2}>0, \\
& 1 \leq \lambda_{1}^{*}<1+\frac{\lambda_{2}^{*}}{\Gamma(2-q)}, \lambda_{2}^{*}>0 .
\end{aligned}
$$

Define the operator $T: X \times X \rightarrow X \times X$ as

$T(u, v)(t)$

$$
\begin{aligned}
= & \left(T_{1}(u, v)(t), T_{2}(u, v)(t)\right) \\
= & \left(\int_{0}^{1} G_{\alpha}(t, s) f_{1}\left(s, u(s), v(s),{ }^{c} D_{0+}^{p} u(s),{ }^{c} D_{0+}^{q} v(s)\right) d s,\right. \\
& \left.\int_{0}^{1} G_{\beta}(t, s) f_{2}\left(s, u(s), v(s),{ }^{c} D_{0+}^{p} u(s),{ }^{c} D_{0+}^{q} v(s)\right) d s\right),
\end{aligned}
$$

which implies

$$
\begin{aligned}
&(T(u, v))^{\prime}(t):=\left(\left(T_{1}(u, v)\right)^{\prime}(t),\left(T_{2}(u, v)\right)^{\prime}(t)\right) \\
&= \int_{0}^{t} \frac{(t-s)^{\alpha-2}}{\Gamma(\alpha-1)} f_{1}\left(s, u(s),{ }^{c} D_{0+}^{p} u(s),{ }^{c} D_{0+}^{q} v(s)\right) d s \\
&-\frac{1}{\Lambda \Gamma(\alpha)} \int_{0}^{1}(1-s)^{\alpha-1} f_{1}\left(s, u(s),{ }^{c} D_{0+}^{p} u(s),{ }^{c} D_{0+}^{q} v(s)\right) d s \\
&-\frac{\lambda_{2}}{\Lambda \Gamma(\alpha-p)} \int_{0}^{1}(1-s)^{\alpha-p-1} f_{1}\left(s, u(s),{ }^{c} D_{0+}^{p} u(s),{ }^{c}\right. \\
& \int_{0}^{t} \frac{(t-s)^{\beta-2}}{\Gamma(\beta-1)} f_{2}\left(s, u(s),{ }^{c} D_{0+}^{p} u(s),{ }_{0+}^{c} D_{0+}^{q} v(s)\right) d s \\
&- \frac{1}{\Lambda^{*} \Gamma(\beta)} \int_{0}^{1}(1-s)^{\beta-1} f_{2}\left(s, u(s),{ }^{c} D_{0+}^{p} u(s),{ }^{c} D_{0+}^{q} v(s)\right) d s \\
&- \frac{\lambda_{2}^{*}}{\Lambda^{*} \Gamma(\beta-p)} \int_{0}^{1}(1-s)^{\beta-p-1} f_{2}\left(s, u(s),{ }^{c} D_{0+}^{p} u(s),{ }^{c}\right. \\
&\left.\left.D_{0+}^{q} v(s)\right) d s\right) .
\end{aligned}
$$

Lemma 3.1. The operator $T: X \times X \rightarrow X \times X$ is completely continuous.

Proof. Firstly, we show that the operator $T: X \times X$ $\rightarrow X \times X$ is continuous. 
For $0<p<1,\left\{u_{n}, v_{n}\right\} \subseteq X \times X$ such that $\left(u_{n}, v_{n}\right) \rightarrow\left(u_{0}, v_{0}\right)$ in $X \times X$, we have

$$
\begin{aligned}
& \left.\max _{t \in[0,1]}\right|^{c} D_{0+}^{p} u_{n}(t)-{ }^{c} D_{0+}^{p} u_{0}(t) \mid \\
= & \max _{t \in[0,1]} \mid \frac{1}{\Gamma(1-p)} \int_{0}^{t}(t-s)^{-p} u_{n}^{\prime}(s) d s-\frac{1}{\Gamma(1-p)} \\
& \quad \times \int_{0}^{t}(t-s)^{-p} u_{0}(s) d s \mid \\
= & \max _{t \in[0,1]}\left|\frac{1}{\Gamma(1-p)} \int_{0}^{t}(t-s)^{-p}\left[u_{n}^{\prime}(s)-u_{0}(s)\right] d s\right| \\
= & \frac{1}{\Gamma(2-p)} \max _{t \in[0,1]}\left|u^{\prime}{ }_{n}(t)-u_{0}(t)\right| \\
= & \frac{1}{\Gamma(2-p)}\left\|u_{n}-u_{0}\right\|_{X} .
\end{aligned}
$$

By $\left\|u_{n}-u_{0}\right\|_{X} \rightarrow 0$, we get the sequence ${ }^{c} D_{0+}^{p} u_{n}(t)$ converges uniformly on $[0,1]$ with

$$
\lim _{n \rightarrow \infty}^{c} D_{0+}^{p} u_{n}(t)={ }^{c} D_{0+}^{p} u_{0}(t) .
$$

Similarly, by $\left\|v_{n}-v_{0}\right\|_{X} \rightarrow 0$, we get the sequence ${ }^{c} D_{0+}^{q} v_{n}(t)$ converges uniformly on $[0,1]$ with $\lim _{n \rightarrow \infty}^{c} D_{0+}^{q} v_{n}(t)={ }^{c} D_{0+}^{q} v_{0}(t)$.

Since

$$
\begin{aligned}
& \left\|T\left(u_{n}, v_{n}\right)-T\left(u_{0}, v_{0}\right)\right\|_{X \times X} \\
= & \max _{t \in[0,1]}\left|T_{1}\left(u_{n}, v_{n}\right)(t)-T_{1}\left(u_{0}, v_{0}\right)(t)\right| \\
& +\max _{t \in[0,1]}\left|\left(T_{1}\left(u_{n}, v_{n}\right)\right)^{\prime}(t)-\left(T_{1}\left(u_{0}, v_{0}\right)\right)^{\prime}(t)\right| \\
& +\max _{t \in[0,1]}\left|T_{2}\left(u_{n}, v_{n}\right)(t)-T_{2}\left(u_{0}, v_{0}\right)(t)\right| \\
& +\max _{t \in[0,1]}\left|\left(T_{2}\left(u_{n}, v_{n}\right)\right)^{\prime}(t)-\left(T_{2}\left(u_{0}, v_{0}\right)\right)^{\prime}(t)\right| .
\end{aligned}
$$

Combining (3.3),(3.4) with the continuity of $f_{1}, f_{2}$, we can get

$$
\left\|T\left(u_{n}, v_{n}\right)-T\left(u_{0}, v_{0}\right)\right\|_{X \times X} \rightarrow 0,\left(\left(u_{n}, v_{n}\right) \rightarrow\left(u_{0}, v_{0}\right)\right) .
$$

Thus $T$ is continuous in $X \times X$.

Let $\Omega \subset X \times X$ be bounded. Then there exist positive constants $L_{i}>0$ such that

$$
\begin{aligned}
& \left|f_{i}\left(t, u(t), v(t),{ }^{c} D_{0+}^{p} u(t),{ }^{c} D_{0+}^{q} v(t)\right)\right| \leq L_{i}(i=1,2) \\
& \forall(u, v) \in \Omega .
\end{aligned}
$$

Thus, for any $(u, v) \in \Omega$, we have

$$
\begin{aligned}
&\left|T_{1}(u, v)(t)\right| \leq \frac{1}{\Gamma(\alpha)} \int_{0}^{t}(t-s)^{\alpha-1} \mid f_{1}\left(s, u(s), v(s),{ }^{c} D_{0+}^{p} u(s),{ }^{c}\right. \\
&\left.D_{0+}^{q} v(s)\right) \mid d s \\
& \frac{\left|\lambda_{1}-t\right|}{\Lambda \Gamma(\alpha)} \int_{0}^{1}(1-s)^{\alpha-1} \mid f_{1}\left(s, u(s), v(s),{ }^{c} D_{0+}^{p} u(s),{ }^{c}\right. \\
&+\frac{\lambda_{2}\left|\lambda_{1}-t\right|}{\Lambda \Gamma(\alpha-p)} \int_{0}^{1}(1-s)^{\alpha-p-1} \mid f_{1}\left(s, u(s), v(s),{ }^{c} D_{0+}^{p} u(s),{ }^{c}\right. \\
&\left.D_{0+}^{q} v(s)\right) \mid d s
\end{aligned}
$$

$$
\begin{aligned}
\leq & \frac{L_{1}}{\Gamma(\alpha+1)}+\frac{L_{1} \lambda_{1}}{\Lambda \Gamma(\alpha+1)}+\frac{\lambda_{1} \lambda_{2} L_{1}}{\Lambda \Gamma(\alpha-p+1)} \\
= & \frac{L_{1}\left(\Lambda+\lambda_{1}\right)}{\Lambda \Gamma(\alpha+1)}+\frac{\lambda_{1} \lambda_{2} L_{1}}{\Lambda \Gamma(\alpha-p+1)} \\
\left|\left(T_{1}(u, v)\right)^{\prime}(t)\right| \leq & \frac{1}{\Gamma(\alpha-1)} \int_{0}^{t}(t-s)^{\alpha-2} \mid f_{1}\left(s, u(s), v(s),{ }^{c}\right. \\
& +\frac{1}{\Lambda \Gamma(\alpha)} \int_{0}^{1}(1-s)^{\alpha-1} \mid f_{1}\left(s, u(s), v(s),{ }^{c}\right. \\
& +\frac{\lambda_{2}}{\Lambda \Gamma(\alpha-p)} \int_{0}^{1}(1-s)^{\alpha-p-1}\left|f_{1}\left(s, u(s), v(s),{ }^{c}{ }^{c} D_{0+}^{q} v(s)\right)\right| d s \\
\leq & \frac{L_{1}}{\Gamma(\alpha)}+\frac{\left.D_{0+}^{p} u(s),{ }^{c} D_{0+}^{q} v(s)\right) \mid d s}{\Lambda \Gamma(\alpha+1)}+\frac{L_{1}{ }^{q} L_{1}}{\Lambda \Gamma(\alpha-p+1)} \\
= & \frac{L_{1}(\Lambda \alpha+1)}{\Lambda \Gamma(\alpha+1)}+\frac{\lambda_{2} L_{1}}{\Lambda \Gamma(\alpha-p+1)} .
\end{aligned}
$$

Hence

$\left\|T_{1}(u, v)\right\|_{X} \leq \frac{L_{1}\left(\Lambda+\lambda_{1}+\Lambda \alpha+1\right)}{\Lambda \Gamma(\alpha+1)}+\frac{\lambda_{2} L_{1}\left(\lambda_{1}+1\right)}{\Lambda \Gamma(\alpha-p+1)}=M_{1} L_{1}$

where $M_{1}$ is given by (3.1).

In the same way, we can verify that

$$
\begin{aligned}
\left\|T_{2}(u, v)\right\|_{X} \leq & \frac{L_{2}\left(\Lambda^{*}+\lambda_{1}^{*}+\Lambda^{*} \alpha+1\right)}{\Lambda^{*} \Gamma(\beta+1)} \\
& +\frac{L_{2} \lambda_{2}^{*}\left(\lambda_{1}^{*}+1\right)}{\Lambda^{*} \Gamma(\beta-q+1)}=M_{2} L_{2}
\end{aligned}
$$

where $M_{2}$ is given by (3.1). Thus,

$$
\|T(u, v)\|_{X \times X} \leq M_{1} L_{1}+M_{2} L_{2}:=M
$$

which implies that the operator $T$ is uniformly bounded.

Next we show that $T$ is equicontinuous.

For any $0 \leq t_{1} \leq t_{2} \leq 1$,

$$
\begin{aligned}
& \left|T_{1}\left(u\left(t_{2}\right), v\left(t_{2}\right)\right)-T_{1}\left(u\left(t_{1}\right), v\left(t_{1}\right)\right)\right| \\
\leq & \int_{0}^{t_{1}} \mid\left[G_{\alpha}\left(t_{2}, s\right)-G_{\alpha}\left(t_{1}, s\right)\right] f_{1}\left(s, u(s), v(s),{ }^{c} D_{0+}^{p} u(s),{ }^{c}\right. \\
& \left.D_{0+}^{q} v(s)\right) \mid d s \\
+ & \int_{t_{1}}^{t_{2}} \mid\left[G_{\alpha}\left(t_{2}, s\right)-G_{\alpha}\left(t_{1}, s\right)\right] f_{1}\left(s, u(s), v(s),{ }^{c} D_{0+}^{p} u(s),{ }^{c}\right. \\
& \left.D_{0+}^{q} v(s)\right) \mid d s \\
+ & \int_{t_{2}}^{1} \mid\left[G_{\alpha}\left(t_{2}, s\right)-G_{\alpha}\left(t_{1}, s\right)\right] f_{1}\left(s, u(s), v(s),{ }^{c} D_{0+}^{p} u(s),{ }^{c}\right. \\
\left.D_{0+}^{q} v(s)\right) \mid d s & L_{1}\left[\frac{t_{2}-t_{1}}{\Lambda \Gamma(\alpha+1)}+\frac{t_{2}^{\alpha}-t_{1}^{\alpha}}{\Gamma(\alpha+1)}+\frac{\lambda_{2}\left(t_{2}-t_{1}\right)}{\Lambda \Gamma(\alpha-p+1)}\right]
\end{aligned}
$$




$$
\begin{aligned}
& \left|\left(T_{1}(u, v)\right)^{\prime}\left(t_{2}\right)-\left(T_{1}(u, v)\right)^{\prime}\left(t_{1}\right)\right| \\
= & \frac{1}{\Gamma(\alpha-1)} \mid \int_{0}^{t_{2}}\left(t_{2}-s\right)^{\alpha-2} f_{1}\left(s, u(s), v(s),{ }^{c} D_{0+}^{p} u(s),{ }^{c}\right. \\
& \left.D_{0+}^{q} v(s)\right) d s \\
& \quad-\int_{0}^{t_{1}}\left(t_{1}-s\right)^{\alpha-2} f_{1}\left(s, u(s), v(s),{ }^{c} D_{0+}^{p} u(s),{ }^{c}\right. \\
& \left.D_{0+}^{q} v(s)\right) d s \mid \\
\leq & \frac{L_{1}}{\Gamma(\alpha-1)}\left|\int_{0}^{t_{2}}\left(t_{2}-s\right)^{\alpha-2} d s-\int_{0}^{t_{1}}\left(t_{1}-s\right)^{\alpha-2} d s\right| \\
\leq & \frac{L_{1}}{\Gamma(\alpha)}\left(t_{2}^{\alpha-1}-t_{1}^{\alpha-1}\right) .
\end{aligned}
$$

Analogously, we can obtain the following inequalities:

$$
\begin{aligned}
& \left|T_{2}\left(u\left(t_{2}\right), v\left(t_{2}\right)\right)-T_{2}\left(u\left(t_{1}\right), v\left(t_{1}\right)\right)\right| \\
\leq & L_{2}\left[\frac{t_{2}-t_{1}}{\Lambda^{*} \Gamma(\beta+1)}+\frac{t_{2}^{\beta}-t_{1}^{\beta}}{\Gamma(\beta+1)}+\frac{\lambda_{2}^{*}\left(t_{2}-t_{1}\right)}{\Lambda^{*} \Gamma(\beta-q+1)}\right], \\
& \left|\left(T_{2}(u, v)\right)^{\prime}\left(t_{2}\right)-\left(T_{2}(u, v)\right)^{\prime}\left(t_{1}\right)\right| \\
\leq & \frac{L_{1}}{\Gamma(\beta)}\left(t_{2}^{\beta-1}-t_{1}^{\beta-1}\right) .
\end{aligned}
$$

Since the functions $t^{\alpha}, t^{\beta}, t^{\alpha-1}, t^{\beta-1}$ are uniformly continuous on the interval $[0,1]$, we can conclude that $T(u, v)$ is equicontinuous on $[0,1]$.

Thus, the operator $T: X \times X \rightarrow X \times X$ is completely continuous. The proof is completed.

Theorem 3.2. Assume that there exist positive constants $c_{i}, d_{i}(i=0,1,2,3,4), c_{0}>0, d_{0}>0, c_{i}, d_{i} \geq 0(i=1$, $2,3,4)$ such that

$$
\begin{gathered}
\forall\left(t, x_{1}, x_{2}, x_{3}, x_{4}\right) \in[0,1] \times R_{+}^{2} \times R^{2}, t \in[0,1], \\
\begin{aligned}
\left|f_{1}\left(t, x_{1}, x_{2}, x_{3}, x_{4}\right)\right| \leq & c_{0}+c_{1}\left|x_{1}\right|+c_{2}\left|x_{2}\right| \\
& +c_{3}\left|x_{3}\right|+c_{4}\left|x_{4}\right|, \\
\left|f_{2}\left(t, x_{1}, x_{2}, x_{3}, x_{4}\right)\right| \leq & d_{0}+d_{1}\left|x_{1}\right|+d_{2}\left|x_{2}\right| \\
& +d_{3}\left|x_{3}\right|+d_{4}\left|x_{4}\right| .
\end{aligned}
\end{gathered}
$$

In addition, assume that

$$
\begin{aligned}
& M_{1} c_{1}+M_{2} d_{1}+\left(M_{1} c_{3}+M_{2} d_{3}\right) \frac{1}{\Gamma(2-p)} \\
< & 1, M_{1} c_{2}+M_{2} d_{2}+\left(M_{1} c_{4}+M_{2} d_{4}\right) \frac{1}{\Gamma(2-q)}<1,
\end{aligned}
$$

where $M_{1}$ and $M_{2}$ are defined by (3.1).

Then the problem (1.1) has at least one positive solution.

Proof. Let us verify that the set

$$
V=\{(u, v) \in X \times X \mid(u, v)=\mu T(u, v), 0 \leq \mu \leq 1\}
$$

is bounded. Let $(u, v) \in V$, then $(u, v)=\mu T(u, v)$. For any $t \in[0,1]$, we have

$$
\begin{aligned}
& |u(t)|=\mu\left|T_{1}(u, v)(t)\right| \\
& \leq \frac{1}{\Gamma(\alpha)} \int_{0}^{t} \mu(t-s)^{\alpha-1} \mid f_{1}\left(s, u(s), v(s),{ }^{c} D_{0+}^{p} u(s),\right. \\
& \left.{ }^{c} D_{0+}^{q} v(s)\right) \mid d s \\
& +\frac{\lambda_{1}-t}{\Lambda \Gamma(\alpha)} \int_{0}^{1} \mu(1-s)^{\alpha-1} \mid f_{1}\left(s, u(s), v(s),{ }^{c} D_{0+}^{p} u(s),\right. \\
& \left.{ }^{c} D_{0+}^{q} v(s)\right) \mid d s \\
& +\frac{\lambda_{2}\left(\lambda_{1}-t\right)}{\Lambda \Gamma(\alpha-p)} \int_{0}^{1} \mu(1-s)^{\alpha-p-1} \mid f_{1}(s, u(s), v(s), \\
& \left.{ }^{c} D_{0+}^{p} u(s),{ }^{c} D_{0+}^{q} v(s)\right) \mid d s \\
& \leq\left[c_{0}+c_{1}|u(t)|+c_{2}|v(t)|+\left.c_{3}\right|^{c} D_{0+}^{p} u(t) \mid\right. \\
& \left.+\left.c_{4}\right|^{c} D_{0+}^{q} v(t) \mid\right] \times\left(\frac{\Lambda+\lambda_{1}}{\Lambda \Gamma(\alpha+1)}+\frac{\lambda_{1} \lambda_{2}}{\Lambda \Gamma(\alpha-p+1)}\right) \\
& \leq\left[c_{0}+c_{1}\|u\|_{X}+c_{2}\|v\|_{X}+c_{3} \frac{1}{\Gamma(2-p)}\|u\|_{X}\right. \\
& \left.+c_{4} \frac{1}{\Gamma(2-q)}\|v\|_{X}\right] \\
& \times\left(\frac{\Lambda+\lambda_{1}}{\Lambda \Gamma(\alpha+1)}+\frac{\lambda_{1} \lambda_{2}}{\Lambda \Gamma(\alpha-p+1)}\right), \\
& \left|u^{\prime}(t)\right|=\mu\left|\left(T_{1}(u, v)\right)^{\prime}(t)\right| \\
& =\frac{1}{\Gamma(\alpha-1)} \int_{0}^{t} \mu(t-s)^{\alpha-2} \mid f_{1}\left(s, u(s), v(s),{ }^{c} D_{0+}^{p} u(s),\right. \\
& \left.{ }^{c} D_{0+}^{q} v(s)\right) \mid d s \\
& -\frac{1}{\Lambda \Gamma(\alpha)} \int_{0}^{1} \mu(1-s)^{\alpha-1} \mid f_{1}\left(s, u(s), v(s),{ }^{c} D_{0+}^{p} u(s),\right. \\
& \left.{ }^{c} D_{0+}^{q} v(s)\right) \mid d s \\
& \frac{\lambda_{2}}{\Lambda \Gamma(\alpha-p)} \int_{0}^{1}(1-s)^{\alpha-p-1} \mid f_{1}(s, u(s), v(s), \\
& \left.{ }^{c} D_{0+}^{p} u(s),{ }^{c} D_{0+}^{q} v(s)\right) \mid d s \\
& \leq\left[c_{0}+c_{1}|u(t)|+c_{2}|v(t)|+\left.c_{3}\right|^{c}\right. \\
& \left.D_{0+}^{p} u(t)\left|+c_{4}\right|^{c} D_{0+}^{q} v(t) \mid\right] \\
& \left(\frac{\Lambda \alpha+1}{\Lambda \Gamma(\alpha+1)}+\frac{\lambda_{2}}{\Lambda \Gamma(\alpha-p+1)}\right) \\
& \leq\left[c_{0}+c_{1}\|u\|_{X}+c_{2}\|v\|_{X}+c_{3} \frac{1}{\Gamma(2-p)}\|u\|_{X}\right. \\
& \left.+c_{4} \frac{1}{\Gamma(2-q)}\|v\|_{X}\right] \\
& \times\left(\frac{\Lambda \alpha+1}{\Lambda \Gamma(\alpha+1)}+\frac{\lambda_{2}}{\Lambda \Gamma(\alpha-p+1)}\right) .
\end{aligned}
$$

Hence,

$$
\begin{aligned}
\|u\|_{X} \leq & M_{1}\left[c_{0}+c_{1}\|u\|_{X}+c_{2}\|v\|_{X}\right. \\
& \left.+c_{3} \frac{1}{\Gamma(2-p)}\|u\|_{X}+c_{4} \frac{1}{\Gamma(2-q)}\|v\|_{X}\right]
\end{aligned}
$$


where $M_{1}$ is defined by (3.1). Similarly, we can get

$\|v\|_{X} \leq M_{2}\left[d_{0}+d_{1}\|u\|_{X}+d_{2}\|v\|_{X}\right.$

$$
\left.+d_{3} \frac{1}{\Gamma(2-p)}\|u\|_{X}+d_{4} \frac{1}{\Gamma(2-q)}\|v\|_{X}\right]
$$

where $M_{2}$ is defined by (3.1). Combining (3.8) with (3.9), we obtain

$$
\begin{aligned}
& \|(u, v)\|_{X \times X} \\
= & \|u\|_{X}+\|v\|_{X} \\
\leq & {\left[M_{1} c_{1}+M_{2} d_{1}+\left(M_{1} c_{3}+M_{2} d_{3}\right) \frac{1}{\Gamma(2-p)}\right]\|u\|_{X} } \\
& +\left[M_{1} c_{2}+M_{2} d_{2}+\left(M_{1} c_{4}+M_{2} d_{4}\right) \frac{1}{\Gamma(2-q)}\right]\|v\|_{X} \\
& +M_{1} c_{0}+M_{2} d_{0} \\
\leq & M_{3}\left(\|u\|_{X}+\|v\|_{X}\right)+M_{1} c_{0}+M_{2} d_{0} .
\end{aligned}
$$

As a result

$$
\|(u, v)\|_{X \times X} \leq \frac{M_{1} c_{0}+M_{2} d_{0}}{1-M_{3}},
$$

for any $t \in[0,1]$, where $M_{3}$ is given by (3.2). So the set $V$ is bounded.

Thus, by Lemma 2.7, the operator $T$ has at least one fixed point. Hence the problem (1.1) has at least one positive solution. The proof is completed.

Theorem 3.3. Assume that both $f_{1}$ and

$$
f_{2}:[0,1] \times R_{+}^{2} \times R^{2} \rightarrow R
$$

are continuous functions and there exist constants $n_{i}, n_{i}^{*} \geq 0(i=1,2,3,4)$, such that

$$
\begin{aligned}
\forall t \in[0,1], & \left(u_{1}, u_{2}, u_{3}, u_{4}\right) \in R_{+}^{2} s R^{2},\left(v_{1}, v_{2}, v_{3}, v_{4}\right) \in R_{+}^{2} \times R^{2}, \\
& \left|f_{1}\left(t, u_{1}, u_{2}, u_{3}, u_{4}\right)-f_{1}\left(t, v_{1}, v_{2}, v_{3}, v_{4}\right)\right| \\
\leq & n_{1}\left|u_{1}-v_{1}\right|+n_{2}\left|u_{2}-v_{2}\right|+n_{3}\left|u_{3}-v_{3}\right| \\
& +n_{4}\left|u_{4}-v_{4}\right| \\
& \left|f_{2}\left(t, u_{1}, u_{2}, u_{3}, u_{4}\right)-f_{2}\left(t, v_{1}, v_{2}, v_{3}, v_{4}\right)\right| \\
\leq & n_{1}^{*}\left|u_{1}-v_{1}\right|+n_{2}^{*}\left|u_{2}-v_{2}\right|+n_{3}^{*}\left|u_{3}-v_{3}\right| \\
& +n_{4}^{*}\left|u_{4}-v_{4}\right| .
\end{aligned}
$$

In addition, assume that

$$
\begin{aligned}
& n_{1}+n_{2}+n_{3} \frac{1}{\Gamma(2-p)}+n_{4} \frac{1}{\Gamma(2-q)} \leq \frac{1}{4 M_{1}}, \\
& n_{1}^{*}+n_{2}^{*}+n_{3}^{*} \frac{1}{\Gamma(2-p)}+n_{4}^{*} \frac{1}{\Gamma(2-q)} \leq \frac{1}{4 M_{2}} .
\end{aligned}
$$

where $M_{1}, M_{2}$ are defined by (3.1). Then the problem (1.1) has a unique solution.

Proof. Define $\sup _{t \in[0,1]}\left|f_{1}(t, 0,0,0,0)\right|=N_{1}<\infty$,

$$
\sup _{t \in[0,1]}\left|f_{2}(t, 0,0,0,0)\right|=N_{2}<\infty \text {, }
$$

such that $r \geq 4 \max \left\{M_{1} N_{1}, M_{2} N_{2}\right\}$. We show that $T B_{r} \subset B_{r}$, where $B_{r}=\{(u, v) \in X \times X\|(u, v)\| \leq r\}$.

For $(u, v) \in B_{r}$, we have

$$
\left|T_{1}(u, v)(t)\right|
$$

$\leq \frac{1}{\Gamma(\alpha)} \int_{0}^{t}(t-s)^{\alpha-1}\left|f_{1}\left(s, u(s), v(s),{ }^{c} D_{0+}^{p} u(s),{ }^{c} D_{0+}^{q} v(s)\right)\right| d s$ $+\frac{\left|\lambda_{1}-t\right|}{\Lambda \Gamma(\alpha)} \int_{0}^{1}(1-s)^{\alpha-1} \mid f_{1}\left(s, u(s), v(s),{ }^{c} D_{0+}^{p} u(s)\right.$,

$$
\left.{ }^{c} D_{0+}^{q} v(s)\right) \mid d s
$$

$$
+\frac{\lambda_{2}\left|\lambda_{1}-t\right|}{\Lambda \Gamma(\alpha-p)} \int_{0}^{1}(1-s)^{\alpha-p-1} \mid f_{1}(s, u(s), v(s),
$$

$\left.{ }^{c} D_{0+}^{p} u(s),{ }^{c} D_{0+}^{q} v(s)\right) \mid d s$

$\leq \frac{1}{\Gamma(\alpha)} \int_{0}^{t}(t-s)^{\alpha-1}\left[\mid f_{1}\left(s, u(s), v(s),{ }^{c} D_{0+}^{p} u(s),{ }^{c} D_{0+}^{q} v(s)\right)\right.$

$\left.-f_{1}(s, 0,0,0,0)|+| f_{1}(s, 0,0,0,0) \mid\right] d s$

$+\frac{\left|\lambda_{1}-t\right|}{\Lambda \Gamma(\alpha)} \int_{0}^{1}(1-s)^{\alpha-1}\left[\mid f_{1}\left(s, u(s), v(s),{ }^{c} D_{0+}^{p} u(s)\right.\right.$,

$\left.\left.{ }^{c} D_{0+}^{q} v(s)\right)-f_{1}(s, 0,0,0,0)|+| f_{1}(s, 0,0,0,0) \mid\right] d s$

$+\frac{\lambda_{2}\left|\lambda_{1}-t\right|}{\Lambda \Gamma(\alpha-p)} \int_{0}^{1}(1-s)^{\alpha-p-1}\left[\mid f_{1}\left(s, u(s), v(s),{ }^{c} D_{0+}^{p} u(s)\right.\right.$,

$\left.\left.{ }^{c} D_{0+}^{q} v(s)\right)-f_{1}(s, 0,0,0,0)|+| f_{1}(s, 0,0,0,0) \mid\right] d s$

$\leq\left[\left(n_{1}+n_{2}+n_{3} \frac{1}{\Gamma(2-p)}+n_{4} \frac{1}{\Gamma(2-q)}\right) r+N_{1}\right]$

$$
\times\left(\frac{\left(\Lambda+\lambda_{1}\right)}{\Lambda \Gamma(\alpha+1)}+\frac{\lambda_{1} \lambda_{2}}{\Lambda \Gamma(\alpha-p+1)}\right),
$$

$$
\left|\left(T_{1}(u, v)\right)^{\prime}(t)\right|
$$

$$
\leq \frac{1}{\Gamma(\alpha-1)} \int_{0}^{t}(t-s)^{\alpha-2} \mid f_{1}\left(s, u(s), v(s),{ }^{c} D_{0+}^{p} u(s),\right.
$$$$
\left.{ }^{c} D_{0+}^{q} v(s)\right) \mid d s
$$

$$
+\frac{1}{\Lambda \Gamma(\alpha)} \int_{0}^{1}(1-s)^{\alpha-1} \mid f_{1}\left(s, u(s), v(s),{ }^{c} D_{0+}^{p} u(s),\right.
$$

$\left.{ }^{c} D_{0+}^{q} v(s)\right) \mid d s$

$$
+\frac{\lambda_{2}}{\Lambda \Gamma(\alpha-p)} \int_{0}^{1}(1-s)^{\alpha-p-1} \mid f_{1}(s, u(s), v(s),
$$

$$
\left.{ }^{c} D_{0+}^{p} u(s),{ }^{c} D_{0+}^{q} v(s)\right) \mid d s
$$

$\leq \frac{1}{\Gamma(\alpha-1)} \int_{0}^{t}(t-s)^{\alpha-2}\left[\mid f_{1}\left(s, u(s), v(s),{ }^{c} D_{0+}^{p} u(s)\right.\right.$,

$\left.\left.{ }^{c} D_{0+}^{q} v(s)\right)-f_{1}(s, 0,0,0,0)|+| f_{1}(s, 0,0,0,0) \mid\right] d s$

$+\frac{1}{\Lambda \Gamma(\alpha)} \int_{0}^{1}(1-s)^{\alpha-1}\left[\mid f_{1}\left(s, u(s), v(s),{ }^{c} D_{0+}^{p} u(s)\right.\right.$,

$\left.\left.{ }^{c} D_{0+}^{q} v(s)\right)-f_{1}(s, 0,0,0,0)|+| f_{1}(s, 0,0,0,0) \mid\right] d s$

$+\frac{\lambda_{2}}{\Lambda \Gamma(\alpha-p)} \int_{0}^{1}(1-s)^{\alpha-p-1}\left[\mid f_{1}(s, u(s), v(s)\right.$,

$\left.{ }^{c} D_{0+}^{p} u(s),{ }^{c} D_{0+}^{q} v(s)\right)-f_{1}(s, 0,0,0,0) \mid$ 


$$
\begin{aligned}
& \left.+\left|f_{1}(s, 0,0,0,0)\right|\right] d s \\
\leq & {\left[\left(n_{1}+n_{2}+n_{3} \frac{1}{\Gamma(2-p)}+n_{4} \frac{1}{\Gamma(2-q)}\right) r+N_{1}\right] } \\
& \times\left(\frac{(\Lambda \alpha+1)}{\Lambda \Gamma(\alpha+1)}+\frac{\lambda_{2}}{\Lambda \Gamma(\alpha-p+1)}\right) .
\end{aligned}
$$

Hence

$$
\begin{aligned}
& \left\|T_{1}(u, v)(t)\right\|_{X} \\
\leq & {\left[\left(n_{1}+n_{2}+n_{3} \frac{1}{\Gamma(2-p)}+n_{4} \frac{1}{\Gamma(2-q)}\right) r+N_{1}\right] M_{1} \leq \frac{r}{2} . }
\end{aligned}
$$

In the same way, we can obtain that

$$
\begin{aligned}
& \left\|T_{2}(u, v)(t)\right\|_{X} \\
\leq & {\left[\left(n_{1}^{*}+n_{2}^{*}+n_{3}^{*} \frac{1}{\Gamma(2-p)}+n_{4}^{*} \frac{1}{\Gamma(2-q)}\right) r+N_{2}\right] M_{2} \leq \frac{r}{2} . }
\end{aligned}
$$

Consequently, $\|T(u, v)(t)\|_{X \times X} \leq r$. Now for $\left(u_{2}, v_{2}\right)$, $\left(u_{1}, v_{1}\right) \in X \times X$ and for any $t \in[0,1]$, we get

$$
\begin{aligned}
& \left|T_{1}\left(u_{2}, v_{2}\right)(t)-T_{1}\left(u_{1}, v_{1}\right)(t)\right| \\
\leq & \frac{1}{\Gamma(\alpha)} \int_{0}^{t}(t-s){ }^{\alpha-1} \mid f_{1}\left(s, u_{2}(s), v_{2}(s),{ }^{c} D_{0+}^{p} u_{2}(s),\right. \\
& \left.{ }^{c} D_{0+}^{q} v_{2}(s)\right)-f_{1}\left(s, u_{1}(s), v_{1}(s),\right. \\
& +\frac{\left|\lambda_{1}-t\right|}{\Lambda \Gamma(\alpha)} \int_{0}^{1}(1-s)^{\alpha-1} \mid f_{1}\left(s, u_{2}(s), v_{2}(s),\right. \\
& -f_{1}\left(s, u_{1}(s), v_{1}(s),{ }^{c} D_{0+}^{p} u_{1}(s),{ }^{c} D_{0+}^{q} v_{1}(s)\right) \mid d s \\
& +\frac{\lambda_{2}\left|\lambda_{1}-t\right|}{\Lambda \Gamma(\alpha-p)} \int_{0}^{1}(1-s)^{\alpha-p-1} \mid f_{1}\left(s, u_{2}(s), v_{2}(s),\right. \\
& -f_{1}\left(s, u_{1}(s), v_{1}(s),{ }^{c} D_{0+}^{p} u_{1}(s),{ }^{c} D_{0+}^{q} v_{1}(s)\right) \mid d s \\
\leq & \left(\frac{\left(\Lambda+\lambda_{1}\right)}{\Lambda \Gamma(\alpha+1)}+\frac{\lambda_{1} \lambda_{2}}{\Lambda \Gamma(\alpha-p+1)}\right)\left(n_{1}+n_{2}+n_{3} \frac{1}{\Gamma(2-p)}\right. \\
& \left.+n_{4} \frac{1}{\Gamma(2-q)}\right)\left(\left\|u_{2}-u_{1}\right\|+\left\|v_{2}-v_{1}\right\|\right), \\
\mid & \left(T_{1}\left(u_{2}, v_{2}\right)\right)^{\prime}(t)-\left(T_{1}\left(u_{1}, v_{1}\right)\right)^{\prime}(t) \mid \\
\leq & \frac{1}{\Gamma(\alpha-1)} \int_{0}^{t}(t-s){ }^{\alpha-2} \mid f_{1}\left(s, u_{2}(s), v_{2}(s),{ }^{c} D_{0+}^{p} u_{2}(s),\right. \\
& +\frac{1}{\Lambda \Gamma(\alpha)} \int_{0}^{1}(1-s)^{\alpha-1} \mid f_{1}\left(s, u_{2}(s), v_{2}(s),\right. \\
\left.{ }^{c} D_{0+}^{q} v_{2}(s)\right)-f_{1}\left(s, u_{1}(s), v_{1}(s),\right. & \left.\left.{ }^{c} D_{1}^{p} u_{0+}^{q}\left(s, v_{1}(s)\right) \mid{ }^{c} D_{0+}^{q} v_{2}(s)\right), v_{1}(s),{ }_{0+}^{p} D_{0+}^{p} u_{1}(s),{ }^{q} D_{0+}^{q} v_{1}(s)\right) \mid d s
\end{aligned}
$$

$$
\begin{aligned}
& +\frac{\lambda_{2}}{\Lambda \Gamma(\alpha-p)} \int_{0}^{1}(1-s)^{\alpha-p-1} \mid f_{1}\left(s, u_{2}(s), v_{2}(s),\right. \\
& -f_{1}\left(s, u_{1}(s), v_{1}(s),{ }^{c} D_{0+}^{p} u_{1}(s),{ }^{c} D_{0+}^{p} u_{0+}^{q} v_{1}(s),{ }^{c} D_{0+}^{q} v_{2}(s)\right) \mid d s \\
& \leq\left(\frac{(\Lambda \alpha+1)}{\Lambda \Gamma(\alpha+1)}+\frac{\lambda_{2}}{\Lambda \Gamma(\alpha-p+1)}\right) \\
& \times\left(n_{1}+n_{2}+n_{3} \frac{1}{\Gamma(2-p)}+n_{4} \frac{1}{\Gamma(2-q)}\right) \\
& \times\left(\left\|u_{2}-u_{1}\right\|+\left\|v_{2}-v_{1}\right\|\right) .
\end{aligned}
$$

Hence

$$
\begin{aligned}
& \left\|T_{1}\left(u_{2}, v_{2}\right)(t)-T_{1}\left(u_{1}, v_{1}\right)(t)\right\|_{X} \\
\leq & M_{1}\left(n_{1}+n_{2}+n_{3} \frac{1}{\Gamma(2-p)}+n_{4} \frac{1}{\Gamma(2-q)}\right) \\
& \times\left(\left\|u_{2}-u_{1}\right\|_{X}+\left\|v_{2}-v_{1}\right\|_{X}\right) .
\end{aligned}
$$

Similarly to the above discussion, we can obtain

$$
\begin{aligned}
& \left\|T_{2}\left(u_{2}, v_{2}\right)(t)-T_{2}\left(u_{1}, v_{1}\right)(t)\right\|_{X} \\
\leq & M_{2}\left(n_{1}^{*}+n_{2}^{*}+n_{3}^{*} \frac{1}{\Gamma(2-p)}+n_{4}^{*} \frac{1}{\Gamma(2-q)}\right) \\
& \times\left(\left\|u_{2}-u_{1}\right\|_{X}+\left\|v_{2}-v_{1}\right\|_{X}\right) .
\end{aligned}
$$

As a result

$$
\begin{aligned}
& \left\|T\left(u_{2}, v_{2}\right)(t)-T\left(u_{1}, v_{1}\right)(t)\right\|_{X \times X} \\
\leq & {\left[M_{1}\left(n_{1}+n_{2}+n_{3} \frac{1}{\Gamma(2-p)}+n_{4} \frac{1}{\Gamma(2-q)}\right)\right.} \\
& \left.+M_{2}\left(n_{1}^{*}+n_{2}^{*}+n_{3}^{*} \frac{1}{\Gamma(2-p)}+n_{4}^{*} \frac{1}{\Gamma(2-q)}\right)\right] \\
& \times\left(\left\|u_{2}-u_{1}\right\|_{X}+\left\|v_{2}-v_{1}\right\|_{X}\right) .
\end{aligned}
$$

Since

$$
\begin{aligned}
& {\left[M_{1}\left(n_{1}+n_{2}+n_{3} \frac{1}{\Gamma(2-p)}+n_{4} \frac{1}{\Gamma(2-q)}\right)\right.} \\
& \left.+M_{2}\left(n_{1}^{*}+n_{2}^{*}+n_{3}^{*} \frac{1}{\Gamma(2-p)}+n_{4}^{*} \frac{1}{\Gamma(2-q)}\right)\right]<1
\end{aligned}
$$

therefore $T$ is a contraction operator.

Thus the conclusion of the theorem holds by using the fixed point theorem of contraction mapping principle. The proof is completed.

\section{Acknowledgements}

This work was financially supported by NNSF of China Grant No.11271087, and No.61263006, Guangxi Scientific Experimental ( China- ASEAN Research ) Centre No.20120116.

\section{REFERENCES}

[1] R. P. Agarwal, D. O’Regan and S. StaneK, "Positive Solutions of the Boundary Value Problem for Nonlinear Fractional Differential Equations,” Journal of Mathatical 
Analysis and Application, Vol. 371, No. 1, 2010, pp. 57-68.

doi: 10.1016/j.jmaa.2010.04.034

[2] B. Ahmad and J. J. Nieto, "Existence Results for a Couple System of Nonlinear Fractional Differential Equations with Three-Point Boundary Conditions," Computers\& Mathematics with Applications, Vol. 58, No. 9, 2009, pp. 1838-1843.

doi: 10.1016/j.camwa.2009.07.091

[3] C. Z. Bai and J. X. Fang, "The Existence of a Positive Solution for a Singular Coupled Systems of Nonlinear Fractional Differential Equations,” Applied Mathematics and Computation, Vol. 150, No. 3, 2004, pp. 611-621. doi: 10.1016/S0096-3003(03)00294-7

[4] Z. B. Bai and H. S. Lu, "Positive Solutions of Boundary Value Problems of Nonlinear Fractional Differential Equation,” Journal of Mathematical Analysis and Applications, Vol. 311, No. 2, 2005, pp. 495-505. doi: 10.1016/j.jmaa.2005.02.052

[5] R. A. C. Ferreira, "Positive Solutions for a Class of Boundary Value Problems with Fractional Q-Differences," Computer \& Mathematics with Applications, Vol. 61, 2011, No. 2, pp. 367-373. doi: 10.1016/j.camwa.2010.11.012

[6] M. Feckan, Y. Zhou and J. R. Wang, “On the Concept and Existence of Solution for Impulsive Fractional Differential Equations," Communications in Nonlinear Science and Numerical Simulation, Vol. 17, No. 7, 2012, pp. 3050-3060. doi: 10.1016/j.cnsns.2011.11.017

[7] C. S. Goodrich, "Existence of a Positive Solution to a System of Discrete Fractional Boundary Value Problems," Allied Mathematics and Computation, Vol. 217, No. 9, 2011, pp. 4740-4753. doi: 10.1016/j.amc.2010.11.029

[8] A. A. Kilbas, H. M. Srivastava and J. J. Trujillo, "Theory and Applications of Fractional Differential Equations,” in: North-Holland Mathematics Studies, Vol. 204, Elservier Science B.V., Amsterdam, 2006.

[9] V. Lakshmikantham, S. Leela and J. Vasundhara Devi, “Theory of Fractional Dynamic Systems," Camb. Acad. Publ., Cambridge, 2009.

[10] V. Lakshmikantham and A. S. Vatsala, "Basic Theory of Fractional Differntial Equations," Nonlinear Analysis, Vol. 69, No. 8, 2009, pp. 2677-2682. doi: 10.1016/j.na.2007.08.042

[11] V. Lakshmikantham, "Theory of Fractional Functional Diffrential Equations,” Nonlinear Analysis, Vol. 69, No. 10, 2008, pp. 3337-3343. doi: $10.1016 /$ j.na.2007.09.025

[12] C. F. Li, X. Z. Luo and Y. Zhou, "Existence of Positive Solutions of the Boundary Value Problem for Nonlinear Fracional Differential Equations," Computers and Mathematics with Applications, Vol. 59, No. 3, 2010, pp. 1363-1375. doi: 10.1016/j.camwa.2009.06.029

[13] Z. H. Liu and J. H. Han, "Integral Boundary Value Problems for Fractional Order Integro-differential Equations,”
Dynamic Systems and Applications, Vol. 21, 2012, pp. 535-548.

[14] Z. H. Liu and X. W. Li, "Existence and Uniqueness of Solutions for the Nonlinear Impulsive Fractional Differential equations," Communications in Nonlinear Science and Numerical Simulation, Vol. 18, No. 6, 2013, pp. 1362-1373.

doi: 10.1016/j.cnsns.2012.10.010

[15] Z. H. Liu and X. W. Li, "On the Controllability of Impulsive Fractional Evolution Inclusions in Banach Spaces," Journal of Optimization Theory and Applications, Vol. 156, No. 1, 2013, pp. 167-182. doi: $10.1007 / \mathrm{s} 10957-012-0236-\mathrm{x}$

[16] Z. H. Liu and L. Lu, “A Class of BVPs for Nonlinear Fractional Differential Equations with P-Laplacian Operator,” E. J. Qualitative Theory of Differential Equations, No. 70, 2012, pp. 1-16.

[17] Z. H. Liu and J. H. Sun, "Nonlinear Boundary Value Problems of Fractional Functional Integro-differential Equations," Computers and Mathematics with Applications, Vol. 64, No. 10, 2012, pp. 3228-3234. doi: 10.1016/j.camwa.2012.02.026

[18] Z. H. Liu and J. H. Sun, "Nonlinear Boundary Value Problems of Fractional Differential Systems," Computers and Mathematics with Applications, Vol. 64, No. 4, 2012, pp. 463-475.

doi: 10.1016/j.camwa.2011.12.020

[19] R. Ma and L. Xu, "Existence of Positive Solutions of a Nonlinear Fourth-order Boundary Value Problem," Applied Mathematics Letters, Vol. 23, No. 5, 2010, pp. 537-543. doi: 10.1016/j.aml.2010.01.007

[20] K. S. Miller and B. Ross, "An Introduction to the Fractional Calculus and Fractional Differential Equations," Wiley, New York, 1993.

[21] I. Podlubny, "Fractional Differential Equations,” Academic Press, San Diego, 1999.

[22] D. R. Smart, "Fixed Point Theorems," Cambridge University Press, 1980.

[23] J. Sabatier, O. P. Agrawal, J. A. T. Machado (Eds.), “Advances in Fractional Calculus:Theoretical Developments and Applications in Physics and Engineering," Springer, Dordrecht, 2007. doi: 10.1007/978-1-4020-6042-7

[24] S. G. Samko, A. A. Kilbas and O. I. Marichev, "Fractional Integral and Derivatives,” Theory and Applications, Gordon and Breach, Yverdon, 1993.

[25] J. H. Sun, Y. L. Liu and G. F. Liu, "Existence of Solutions for Fractional Differential Systems with Antiperiodic Boundary Conditions," Computers \& Mathematics with Applications, Vol. 64, No. 6, 2012, pp. 1557-1566. doi: 10.1016/j.camwa.2011.12.083

[26] X. Su, "Boundary Value Problem for a Couple Systems of Nonliear Fractional Differential Equations," Applied Mathematics Letters, Vol. 22, 2009.

[27] J. H. Wang, H. J. Xiang and Z. G. Liu, "Positive Solution to Nonzero Boundary Values Problem for a Coupled System of Nonlinear Fractional Differential Equations," In- 
ternational Journal of Differential Equations, Vol. 2010, 2010, p. 12.

doi: $10.1155 / 2010 / 186928$

[28] X. Xu, D. Jiang and C. Yuan, "Multiple Positive Solutions for the Boundary Value Problem of a Nonlinear Fractional Differential Equation," Nonlinear Analysis, Vol. 71, No. 10, 2009, pp. 4676-4688. doi: 10.1016/j.na.2009.03.030

[29] X. Yang, Z. L. Wei and W. Dong, "Existence of Positive Solutions for the Boundary Value Problem of Nonlinear Fractional Differential Equations," Communications in Nonlinear Science and Numerical Simulation.

[30] X. Yang, Z. Wei and W. Dong, "Existence of Positive Solutions for the Boundary Value Problem of Nonlineear Fractional Differential Equations," Communications in Nonlinear Science and Numerical Simulation, Vol. 17, 2012, pp. 85-92.
[31] S. Zhang, "Positive Solutions for Boundary Value Problems of Nonlinear Fractional Differential Equations," Elec. Journal of Differential Equations, Vol. 36, 2006, pp. 1-12.

[32] Y. G. Zhao, S. R. Sun, Z. L. Han and M. Zhang, "Positive Solutions for Boundary Value Problems of Nonlinear Fractional Differential Equations,” Applied Mathematics and Computations, Vol. 2, No. 17, 2011, pp. 6950-6958. doi: 10.1016/j.amc.2011.01.103

[33] Y. Zhao, S. Sun, Z. Han and Q. Li, "Positive Solutions to Boundary Value Problems of Nonlinear Fractional Differential Equation,” Abstract and Aplied Analysis, Vol. 2011, 2011, p. 16. doi: $10.1155 / 2011 / 390543$

[34] Y. Zhou and F. Jiao, "Nonlocal Cauchy Problem for Fractional Evolution Equations,” Nonlinear Analysis, Vol. 11, 2010, pp. 4465-4475. 